\title{
Apoptosis characterizes immunological failure of HIV infected patients
}

\author{
Marie-José Mhawej $^{\mathrm{a}}$, Damien Ernst ${ }^{\mathrm{b}}$, Raphael Fonteneau ${ }^{\mathrm{b}}$, \\ Claude H. Moog ${ }^{\text {a }}$, Guy-Bart Stan ${ }^{\mathrm{c}}$, \\ ${ }^{\mathrm{a}} I R C C y N$, UMR-CNRS 6597, École Centrale de Nantes, 1 Rue de la Noë-BP \\ 92101 - 44321 Nantes Cedex 03, France. \\ ${ }^{\mathrm{b}}$ University of Liège - Department of Electrical Engineering, Montefiore Institute \\ Building B28, B-4000 Liège, Belgium. \\ c University of Cambridge, Department of Engineering, Control Group, \\ Trumpington Street,Cambridge, CB2 1PZ, United Kingdom.
}

\begin{abstract}
This paper studies the influence of apoptosis in the dynamics of the HIV infection. A new modeling of the healthy CD4 + T-cells activation-induced apoptosis is used. The parameters of this model are identified by using clinical data generated by monitoring patients starting Highly Active Anti-Retroviral Therapy (HAART). The sampling of blood tests is performed to satisfy the constraints of dynamical system parameter identification. The apoptosis parameter, which is inferred from clinical data, is then shown to play a key role in the early diagnosis of immunological failure.
\end{abstract}

Key words: Non linear systems, biomedical systems, identifiability, identification, parameter estimation, apoptosis, HIV, immunological failure, clinical trial.

\section{Introduction}

Infection with HIV typically causes a progressive decay in the functionality and the number of CD4+ T-lymphocytes that may ultimately lead to the lethal Acquired Immune Deficiency Syndrome (AIDS). Since the first identification of the disease in 1981, intensive studies have been carried out to

Email addresses: \{marie-jose.mhawej; claude.moog\}@irccyn.ec-nantes.fr, ernst@montefiore .ulg. ac . be; raphael . fonteneau@ulg. ac.be, gvs22@eng.cam.ac.uk (Guy-Bart Stan). 
understand the fundamental HIV infection mechanisms. Several mechanisms have been involved in the loss of CD4 + T-cells and this topic is one of the most controversial issues in recent AIDS research.

T-cell loss appears to be due to direct destruction by the virus (direct virusinduced cytolisis) or to defective T-cell generation. In 1991, apoptosis, also called programmed cell death, has been suggested as another mechanism responsible for T-cell depletion during the progression of the HIV infection and an extensive body of recent literature is supporting this hypothesis (Pantaleo, G. et al. (1995), Herbein, G. et al. (1998), Marie-Lise Gougeon et al. (1999), Ahr, B. et al. (2004), Badley, A.D. (2005), Yun Yue, F. et al. (2005), Stan, G.B. et al. (2008)). In HIV-infected patients, both infected and uninfected cells undergo exaggerated apoptosis but, remarkably, the vast majority of the cells that undergo apoptosis are uninfected (Vassena, L. et al. (2007)). Furthermore, the level of apoptosis in HIV infected patients is correlated to the levels of circulating CD4 + T-cells and the stage of the disease (Vassena, L. et al. (2007)), which reinforces the idea that apoptosis plays a major role in the death of the lymphocytes.

Mathematical analysis of the HIV/AIDS infection has been actively studied since the middle of the 90's (Perelson, A.S. et al. (1997), Perelson, A.S. et al. (1999)). Nowadays, more specific topics related to the HIV dynamics are being studied, and amongst them, treatment scheduling, resistance emergence and HAART therapy drugs effect. Some particular therapy regimes and/or structured treatment interruptions schemes have been proposed to enhance the immune response and eventually lead to the long-term immunological control of HIV (Wodarz, D. and Nowak, M. (1999), Zurakowski, R. and Teel, R.A. (2006), Chang, H. and Astolfi, A. (2007)). Recently, the correlation existing between treatment interruptions and emergence of resistant viral strains has also been analyzed (Zurakowski, R. and Wodarz, D. (2007), Smith, R.J. (2006)). Concerning the sensitivity of the HIV response to treatment and drug effects, the interested reader is referred to (Smith, R. and Wahl, M. (2004)) and (Khalili, S. and Armaou, A. (2008)).

The mathematical models which are mainly used consist of a set of nonlinear Ordinary Differential Equations (ODEs) which aim at modeling the long-term interaction between the immune system and the virus. These models take into consideration several biological phenomena that influence the infection process, but, to the best of our knowledge, only Stan, G.B. et al. (2008) proposed a model incorporating the activation-induced apoptosis phenomena. In Stan, G.B. et al. (2008), a modification of the model presented in Adams, B.M et al. (2004) is presented and its goal is to propose a simple yet realistic HIV-immune system dynamical interaction model which incorporates the observed activation-induced apoptosis phenomenon and permits the mathematical analysis of its effect on the simulated HIV infection. 
Among a general population of HIV infected patients starting a new therapy, some individuals will eventually undergo a so-called immunological failure. The main contribution of this paper is ti predict such failures based on the identification of an apoptosis parameter. Immunological failure is clinically declared when the amount of CD4 T-cells remains under the level of 200/ $\mathrm{mm}^{3}$ during 6 months of efficient treatment (Delfraissy, JF. (2005); U.S. Dept. Health and Human Services (2006); Ouattara, D. (2005)). An treatment is defined as efficient if it yields a decrease of the viral load below the detectability threshold of 50 copies/ml and keeps the viral load below this limit afterwards. A central paradox for HIV patients who are in immunological failure is that their viral load seems too low to be able to deplete the $\mathrm{CD} 4+$ population by direct killing alone. In this respect, previous studies (Ouattara, D. (2005), Ouattara, D. (2007), Ouattara, D. et al. (2008)) relate immunological failure to a malfunctioning thymus which is unable to produce a sufficient amount of healthy CD $4+$ T-cells. Herein, a different point of view is argued and it is shown that estimation of the value of a specific apoptosis parameter in the transient stage of the infection also allows to diagnose immunological failure. This approach is supported by results derived from real clinical data provided by the CHU de Nantes (Nantes University Hospital) in France.

The outline of this paper is as follows. In Section 2.1 some elementary modeling of the HIV/AIDS dynamics in the form of a 3-Dimensional (3D) continuoustime model is reviewed. In Section 2.2, this 3D model is modified in order to take into account the activation-induced apoptosis phenomenon which directly affects the population of uninfected CD4+ T-cells. Section 2.3 presents a 4-Dimensional continuous-time model which takes into account not only the apoptosis phenomenon, but also the dynamics of cytotoxic T-lymphocytes cells (CTL). In Section 3 the identifiability of the new models is proved and the identification method used for the parameter estimation is described. Working assumptions and the clinical trial design are summarized in Section 4. The main results of this study which concern the relation existing between activation-induced apoptosis and immunological failure are presented in Section 5. Finally, Section 6 offers some concluding remarks as well as some open questions.

\section{Mathematical modeling of apoptosis}

To describe the HIV infection dynamics, several authors attempted to design various mathematical models which are mostly either deterministic (e.g. Perelson, A.S. et al. (1997), Perelson, A.S. et al. (1999), Adams, B.M et al. (2004)) or stochastic (?, Khalili, S. and Armaou, A. (2008)). An extensive overview of the different types of models that have been proposed for treat- 
ing AIDS affected patients using anti-retroviral drug therapies is provided in (Tan, W.Y and Wu, H. (2005)). In particular, recent models tend to have a growing number of variables representing several cell populations involved in the infection process. For example, in (Khalili, S. and Armaou, A. (2008)), the proposed model has 11 differential equations and more than 10 parameters while the basic model described in (Perelson, A.S. et al. (1999)), and (Nowak, M.A (2002)) only has 3 differential equations and 6 parameters. Complex models may be helpful to incorporate some of the phenomena and fine details known about the HIV infection. However, the complexity of such models easily becomes a drawback when considering their rigorous mathematical analysis or if these are to be used for control purposes. Due to the large number of parameters involved and the lack of clinical data, it is often required to simplify models and to restrict their analysis to essential phenomena only. This is the approach chosen in this paper.

\subsection{A Basic 3D model of the HIV infection dynamics}

In this section, a review of the basic 3-Dimensional (3D) continuous-time model of the HIV infection dynamics as described by Perelson, A.S. et al. (1999) and Nowak, M.A (2002) is presented. This model, given hereafter, includes the dynamics of infected CD4 + T-cells, uninfected CD4 + T-cells and virions.

$$
\Sigma_{3 D} \triangleq\left\{\begin{aligned}
\dot{T} & =s-\delta T-\beta T V, \\
\dot{T}^{*} & =\beta T V-\mu T^{*}, \\
\dot{V} & =k T^{*}-c V .
\end{aligned}\right.
$$

In this model, the state variable $T$ (in $\mathrm{CD} 4 / \mathrm{mm}^{3}$ ) represents the amount of uninfected CD $4+$ T-cells, $T^{*}$ (in CD $4 / \mathrm{mm}^{3}$ ) represents the amount of infected $\mathrm{CD} 4+$ T-cells and $V$ (in RNA copies $/ \mathrm{ml}$ ) represents the amount of free virions. Free virus particles infect uninfected T-cells at a rate proportional to both $T$ and $V(\beta T V)$. They are removed from the system at the rate $c V$. In model (1), it is assumed that healthy CD4+ T-cells are produced at a constant rate $s$ (this production typically occurs in the thymus). The terms $\delta T$ and $\mu T^{*}$ represent the death rates of uninfected and infected cells, respectively. The terms $1 / \delta$, $1 / \mu$, and $1 / c$ are the life-times of the uninfected CD4 + T-cells, infected CD4+ T-cells and virions, respectively.

\subsection{A 3D apoptosis modeling}

Apoptosis, also called programmed cell death, is an important biological process that eliminates selected cells for the benefit of the whole organism. The 
"decision" for apoptosis can come from the cell itself, or be induced from its surrounding environment. In the special case of lymphocytes, apoptosis plays an important role in optimizing the immune system by compensating lymphocytes proliferation through the elimination of cells that have become ill or ineffective. When apoptosis is not influenced by the presence of other cells, its effect can be included in the death rate of each cell (represented by the term $-\delta T)$. However, this is not the case for environment dependent apoptosis. As reported in Pantaleo, G. et al. (1995) and Badley, A.D. (2005), lymph nodes of HIV-infected individuals contain a high percentage (with respect to uninfected individuals) of uninfected cells which are in an apoptotic state - that is which are ready to enter an apoptopic process. These cells are prematurely marked for apoptosis due to the presence of several chemical messengers, such as the glycoprotein gp120 (soluble or expressed on the surface of infected cells and virions), proteins (Tat, Nef, Vpr), and also the membrane-bound TNF-alpha on the surface of macrophages (see Herbein, G. et al. (1998), Marie-Lise Gougeon et al. (1999), Zauli, G. et al. (1999) and Stewart, S.A et al. (2000), Wang, J.et al. (2001), Ahr, B. et al. (2004) for more details). For the sake of obtaining a model which is able to qualitatively capture the activation-induced apoptosis phenomenon without becoming too complex for its analysis, it is assumed that all the potential apoptosis-inducing factors are directly correlated with the concentration of HIV-infected CD4 + T-cells. To incorporate this environment-dependent apoptosis into the model given in (1), the healthy T-cells dynamics are modified as follows:

$$
\dot{T}=s-\delta T-\beta T V-\delta_{A} T
$$

where $\delta_{A}$ denotes the death rate of uninfected CD4 + T-cells related to activationinduced apoptosis. $\delta_{A}$ depends on the amount of chemical messengers released by the infected CD4+ T-cells and thus depends on $T^{*}$ :

$$
\dot{T}=s-\delta T-\beta T V-\delta_{A}\left(T^{*}\right) T .
$$

Assuming that the concentration of the chemical messengers inducing apoptosis is proportional to $T^{*}$, and more specifically that $\delta_{A}\left(T^{*}\right)=A T^{*}$, gives:

$$
\dot{T}=s-\delta T-\beta T V-A T^{*} T .
$$

where $A$ is a non negative parameter that represents the "apoptosis rate" (also called apoptosis parameter) of uninfected CD4 + T-cells when in presence of infected CD4+ T-cells. This modelization is directly derived from (Stan, G.B. et al. (2008)) to which the interested reader is referred for de- 
tails and in-depth mathematical analysis. The new model thus reads:

$$
\Sigma_{3 D A} \triangleq\left\{\begin{aligned}
\dot{T} & =s-\delta T-\beta T V-A T T^{*} \\
\dot{T}^{*} & =\beta T V-\mu T^{*}, \\
\dot{V} & =k T^{*}-c V .
\end{aligned}\right.
$$

Equilibrium points of the 3DA model:

By setting $\dot{T}=\dot{T}^{*}=\dot{V}=0$ and solving the corresponding set of equations, it can be shown that there are only 2 equilibrium points for model (2).

(1) HIV free equilibrium:

$T^{\left(e q_{1}\right)}=s / \delta, T^{*\left(e q_{1}\right)}=0, V^{\left(e q_{1}\right)}=0$.

(2) HIV pathological equilibrium:

$$
T^{\left(e q_{2}\right)}=\frac{\mu c}{\beta k}, T^{*\left(e q_{2}\right)}=\frac{s \beta k-\delta \mu c}{\mu(\beta k+A c)}, V^{\left(e q_{2}\right)}=\frac{k(s \beta k-\delta \mu c)}{\mu c(\beta k+A c)} .
$$

Note that an efficient treatment typically leads to undetectable viral loads, which, in the case of this simplified model, can be approximated by a situation where the drug therapy drives the patient to the HIV free equilibrium. It will be shown in Sections 3 and 5 that the proposed model (2) is identifiable and thus can be used to identify the apoptosis parameter $A$ in the transient stage of the therapy response.

For completeness, an elementary dynamics for the cytotoxic T-lymphocytes (CTL) has been added to the HIV infection dynamics considered in (2). This is done in the following Section 2.3. The identifiability of models (2) and (3) is discussed in Section 3. Results are displayed in Section 5.

\section{$2.3 \quad$ A $4 D$ apoptosis modeling}

In this section, a 4-Dimensional HIV model which takes into account the apoptosis and the dynamics of the HIV specific CTL cells is introduced. This model reads:

$$
\Sigma_{4 D A} \triangleq\left\{\begin{aligned}
\dot{T} & =s-\delta T-\beta T V-A T T^{*}, \\
\dot{T}^{*} & =\beta T V-\mu T^{*}-q T^{*} T_{c t l}, \\
\dot{T}_{c t l} & =\lambda+a T T^{*} T_{c t l}-\alpha T_{c t l}, \\
\dot{V} & =k T^{*}-c V .
\end{aligned}\right.
$$

In this model, CTL cells are produced by the thymus at a constant rate $\lambda$. CTL cells are activated when put in contact with infected CD4 + T-cells. 
Furthermore, establishment of the CTL response typically depends on the concentration of CD4+ T-cells (Wodarz, D. and Nowak, M. (1999)). Consequently, one can consider that CTL cells proliferate at a rate $a T T^{*} T_{c t l}$ proportional to $T, T^{*}$ and $T_{c t l}$. The term $\alpha T_{c t l}$ represents the death rate of the CTL cells. CTL cells eliminate infected CD4+ T-cells at a rate $q T^{*} T_{c t l}$. This form of the CTL dynamics has been introduced in (Ouattara, D. (2006)) to which the reader is referred for additional information. Note that the programmed cell death of CTL cells is still a controversial issue and may be a consequence of immune stimulation with no direct link to HIV pathogenesis (Estaquier, J. et al. (1994)). Therefore, apoptosis of CTL cells is not explicitly included in model (3).

Equilibrium points of the $4 D A$ model

Solving the system of equilibrium equations of model (3), it can be shown that it has 3 equilibrium points which are given hereafter.

(1) HIV free equilibrium:

$T^{\left(e q_{1}\right)}=s / \delta, T^{*\left(e q_{1}\right)}=0, T_{c t l}^{\left(e q_{1}\right)}=\lambda / \alpha, V^{\left(e q_{1}\right)}=0$

(2) HIV pathological equilibrium:

$T^{\left(e q_{2}\right)}=Z_{1}, T^{*\left(e q_{2}\right)}=-\frac{c\left(\delta Z_{1}-s\right)}{Z_{1}(\beta k+A c)}, T_{c t l}^{\left(e q_{2}\right)}=-\frac{\mu c-\beta k Z_{1}}{q c}, V^{\left(e q_{2}\right)}=-\frac{k\left(\delta Z_{1}-s\right)}{Z_{1}(\beta k+A c)}$

where

$$
Z_{1}=\frac{-\left(-a c \beta k s+\alpha \beta k A c+\alpha \beta^{2} k^{2}-a c^{2} \mu \delta\right)+\sqrt{\Delta}}{2 a c \beta k \delta}
$$

and

$$
\begin{gathered}
\Delta=\left(-a c \beta k s+\alpha \beta k A c+\alpha \beta^{2} k^{2}-a c^{2} \mu \delta\right)^{2} \\
-4 a c \beta k \delta\left(-\lambda q c \beta k-\lambda q c^{2} A+a c^{2} \mu s-\alpha \mu c^{2} A-\alpha \mu c \beta k\right)
\end{gathered}
$$

(3) The third solution of the system of equilibrium equations corresponds to negative state variables for the average parameters values at steady state. Thus, this equilibrium point has no physical interest.

\section{$3 \quad$ Identifiability and identification method}

\subsection{Identifiability of model (2) and model (3)}

Since available measurements typically contain the total (uninfected and infected) CD4 + T-cells concentration and the concentration of free virions, the output measurements for model (2) are $y_{1}=T+T^{*}$ and $y_{2}=V$. According to the identifiability theory presented in Xia, X. and Moog, C.H. (2003), it is 
shown that the proposed 3D model (2) is algebraically identifiable from the considered output measurements $\left(y_{1}\right.$ and $\left.y_{2}\right)$.

Let $\theta_{1}=s, \theta_{2}=\delta, \theta_{3}=\beta, \theta_{4}=A, \theta_{5}=\mu, \theta_{6}=k, \theta_{7}=c$. The parameters $\theta_{i}$ are identifiable from the measured outputs since

$$
\operatorname{rank}(\Gamma)=\operatorname{rank} \frac{\partial\left(\dot{y}_{1}, \cdots, y_{1}^{(4)}, \ddot{y}_{2}, \cdots, y_{2}^{(4)}\right)}{\partial\left(\theta_{1}, \cdots, \theta_{7}\right)}=7
$$

Straightforward but tedious computations show that the rank condition (4) is satisfied for model (2). In particular, ten measurements at least (5 for $y_{1}$ and 5 for $y_{2}$, i.e 5 blood samples) are required to obtain a first estimate of the 7 parameters of model (2). Considering output measurements $y_{1}=T+T^{*}$, $y_{2}=V$ and $y_{3}=T_{c t l}$, similar computations show that the $4 \mathrm{DA}$ model is also algebraically identifiable. However, 15 measurements at least ( 5 for $y_{1}, 5$ for $y_{2}$ and 5 for $y_{3}$, i.e 5 blood samples) are required to compute the 11 parameters of model (3).

\subsection{The estimation procedure: a Monte-Carlo approach}

The estimation method used in this paper is based on the Monte-Carlo approach introduced in Ouattara, D. (2005). This method is heuristic and relies on the non-linear simplex optimization method which itself is an extension of the simplex optimization approach proposed in Filter, R.A. and Xia, X. (2003). It addresses the problem of high variance of the simplex optimization algorithm with respect to the data set and the initial conditions. This is achieved by drawing a large number of random realizations of initial conditions and taking as estimated values for the parameters the median value of the results obtained for each random initial condition. Its main advantage is that the obtained results are stable and robust with respect to the algorithm initialization values. If the considered model is identifiable, the algorithm returns the parameters estimates and the IQR (interquartile range) of the distribution of each estimated parameter. The IQR is defined as the distance between the $75^{\text {th }}$ percentile and the $25^{\text {th }}$ percentile of the distribution. It measures the dispersion of the results around the real optimum and gives an important information about the confidence on the results. The robustness of this Monte-Carlo method with respect to noisy data was presented in (Ouattara, D. (2006)), (Ouattara, D. (2007)) and (Ouattara, D. et al. (2008)). The estimation algorithm is implemented in a software available at IRCCyN Web software (2007) that allows parameter identification for HIV ODE infection models of individual patients. Estimation results presented in Section 5 were generated with this software. Figure 1 is a typical example of how a specific output of the simulated model fits the experimental data. 
It shows the simulated time evolution of the viral load compared to experimental data for patient 03. In the forthcoming sections, parameter estima-

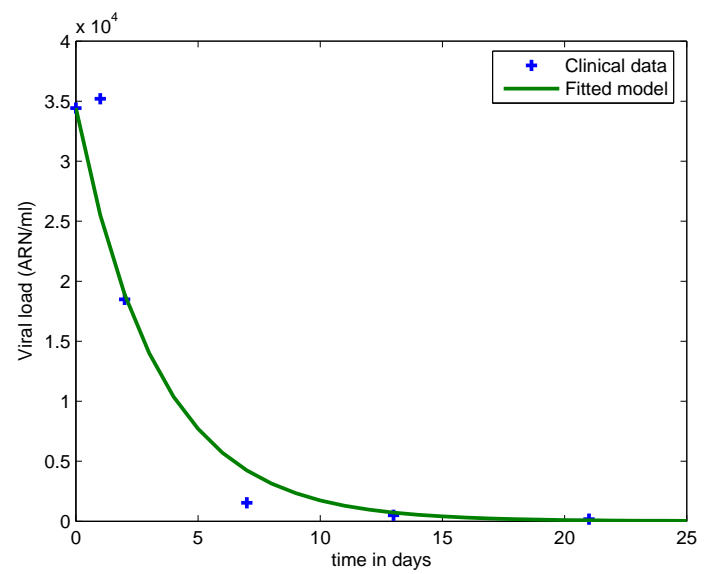

Fig. 1. Simulated viral load compared to clinical data for patient 3 (model 3DA)

tion is done using the algorithm described above. The reader is referred to (Ouattara, D. (2005)), (Ouattara, D. (2006)), (Ouattara, D. (2007)) and (Ouattara, D. et al. (2008)) for more details about this algorithm.

\section{Working context}

\subsection{Assumptions}

Parameters in the mathematical models of the HIV/AIDS infection are related to the immunological or virological status of the patient. For instance, $s$ is the natural production of CD4 T-cells by the thymus. So, a low value for $s$ is representative of a badly damaged immune system. On another hand, a large value for $A$ indicates an important activation-induced apoptosis phenomenon. By estimating the parameters of these models from clinical observations, a numerical quantification of the importance of these biological phenomena becomes possible.

In particular, the aim of this work is to show that immunological failure can be predicted by modeling and analyzing the activation-induced apoptosis phenomenon during the transient stage of the dynamics. Until now, immunological failure was alternatively related to a low value of the parameter $s$ (Ouattara, D. (2005)), which typically means that the depletion of CD4 + T-cells is essentially due to a low production level of healthy CD4+ Tcells by the thymus. Recent literature suggests that the eradication of CD4+ T-cells is mostly due to a strong activation-induced apoptosis phenomenon 
(Pantaleo, G. et al. (1995), Herbein, G. et al. (1998), Marie-Lise Gougeon et al. (1999), Badley, A.D. (2005), Yun Yue, F. et al. (2005), Stan, G.B. et al. (2008)).

In this analysis, it is considered that each of the life-time parameters appearing in $(2)$, i.e. $\delta, \mu$, and $c$, has a fixed, common value for all patients. Indeed, it was shown in (Moog, C.H. et al. (2007)) that the life-time parameters do not vary significantly from one patient to another. Furthermore, a fixed common level of CD4 + T-cells production $s$ is assumed, which amounts to assume that all patient have a healthy thymus. Finally, note that the parameters $\beta$ and $k$ do not a priori reflect any intrinsic success or failure property of the immune system (they characterize the virulence of the infection and potentially include the treatment effects). Therefore, only the value of $A$ computed from the transient response determines the immune system status. Detailed hypotheses and results are presented in Section 5.

\subsection{The EDV05 clinical trial design}

The EDV05 trial was initiated at the CHU of Nantes in February 2005, and 6 patients were included in the first part of this trial. In 2007, 6 other patients were enrolled. These patients did respect a set of enrollment criteria and a specific sampling policy was applied. These are described below.

\subsubsection{Enrollment criteria of patients}

The main conditions for each patient (Female or Male) to be included in the trial were as follows:

- aged over 18 years,

- having been infected by the HIV-1 or HIV-2 type virus,

- not showing any resistance to the administrated treatment,

- no co-infection with HBV (Hepatitis B virus) or HCV (Hepatitis C virus) during the 6 months preceding their inclusion into the trial,

- starting an anti-Retroviral treatment at the beginning of the trial.

All the patients start a treatment (not necessarily the same) at the beginning of the trial $\left(\right.$ Day $\mathrm{D}_{0}$ ). Day $\mathrm{D}_{0}$ is not the same for all the patients. The third condition enables avoiding any viral resistance which is not taken into account in our model. In fact, most of the patients enrolled in this trial, especially the first six, are naive of any treatment at $\mathrm{D}_{0}$. The fourth condition was introduced to avoid dealing with HIV patients whose clinical state is influenced by other infections. Note that 25\% (5\%) of HIV patients, in France, are co-infected by HCV (HBV) (see Delfraissy, JF. (2005)). Table 1 gathers data about the 12 patients enrolled in the trial. 


\begin{tabular}{|c||lr|c|}
\hline Patient ID & \multicolumn{2}{|c|}{ Day $D_{0}$} & Year of birth (age at $\left.D_{0}\right)$ \\
\hline 01 & 14 March & 2005 & $1956(49$ years $)$ \\
02 & 14 March & 2005 & $1967(38$ years $)$ \\
03 & 22 March & 2005 & $1962(43$ years $)$ \\
04 & 22 March & 2005 & $1964(41$ years $)$ \\
05 & 04 April & 2005 & $1967(38$ years $)$ \\
06 & 02 May & 2005 & $1970(35$ years $)$ \\
07 & 12 February & 2007 & 1963 (44 years) \\
08 & 02 April & 2007 & $1971(36$ years $)$ \\
09 & 14 May & 2007 & $1976(31$ years $)$ \\
10 & 04 June & 2007 & $1968(39$ years $)$ \\
11 & 31 December 2007 & $1948(59$ years $)$ \\
12 & 31 December 2007 & $1958(49$ years $)$ \\
\hline
\end{tabular}

Table 1

The 12 patients enrolled in the EDV05 trial. Day $\mathrm{D}_{0}$ is the first day of the trial for the patient (the day of enrollment). The average age at $\mathrm{D}_{0}$ is 41.8 years.

\subsubsection{The data sampling scheduling}

The measured data are the viral load, the total CD4 T-cells count and the total CTL T-cells count. Eleven blood samples were taken during 3 months according to the planning described in Figure 2. The day $\mathrm{D}_{i}$ is equal to the day $\mathrm{D}_{0}+i$. After the initiation of the treatment, at day $\mathrm{D}_{0}$, the viral load drops exponentially with an average time response of 7 days. One explanation for this lies in the fact that the patients do not present any drug resistance. The viral load stabilizes below the detectability level of 50 copies/ml after 3 weeks. As can be observed in Figure 1, the dynamics of the infection is strongly affected by the treatment at the beginning of the trial. Thus, blood samples are taken frequently in the first days of the trial, i.e. in the transient response of the infection dynamics, when measurements contain more information about the dynamics which is not the case when the system stabilizes. More precisely, to collect relevant information about the dynamics, 6 or 7 blood samples are taken during the first 20 days. This enables a first estimation of all the parameters of the 3DA model given in (2). From day 30 to day 90 , only 4 or 5 other samples are taken with a sampling rate of about 15 days. Since the measured viral load values depend on the laboratory protocol used to quantify the RNA copies (Prud'homme, I.T. et al. (1998); Fiches Techniques de la Firme Roche (2003); Berger, A. et al. (2005); Galli R. et al. (2005); Israel Ballard, K. et al. (2005)), it was decided for each patient to perform all the measurements of the viral load at the same time with the same 


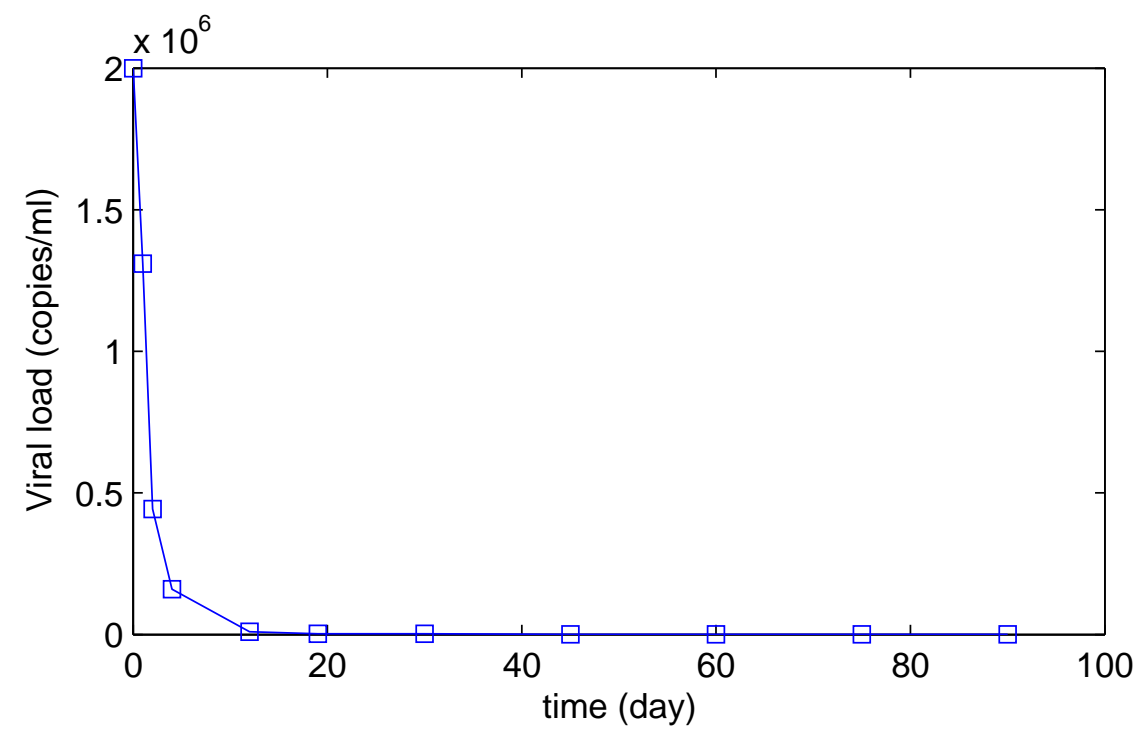

\begin{tabular}{|c||c|c|c|c|c|c|c|c|c|c|c|c|}
\hline \multicolumn{1}{|c||}{ Data } & \multicolumn{10}{|c|}{ Day of measurement } \\
\hline \multicolumn{1}{l||}{} & $\mathrm{P}_{01}$ & $\mathrm{P}_{2}$ & $\mathrm{P} 03$ & $\mathrm{P} 04$ & $\mathrm{P} 05$ & $\mathrm{P} 06$ & $\mathrm{P} 07$ & $\mathrm{P} 08$ & $\mathrm{P} 09$ & $\mathrm{P} 10$ & $\mathrm{P} 11$ & $\mathrm{P}_{12}$ \\
\hline 1 & $\mathrm{D}_{0}$ & $\mathrm{D}_{0}$ & $\mathrm{D}_{0}$ & $\mathrm{D}_{0}$ & $\mathrm{D}_{0}$ & $\mathrm{D}_{0}$ & $\mathrm{D}_{0}$ & $\mathrm{D}_{0}$ & $\mathrm{D}_{0}$ & $\mathrm{D}_{0}$ & $\mathrm{D}_{0}$ & $\mathrm{D}_{0}$ \\
3 & $\mathrm{D}_{1}$ & $\mathrm{D}_{1}$ & $\mathrm{D}_{1}$ & $\mathrm{D}_{1}$ & $\mathrm{D}_{1}$ & $\mathrm{D}_{1}$ & $\mathrm{D}_{4}$ & $\mathrm{D}_{4}$ & $\mathrm{D}_{4}$ & $\mathrm{D}_{4}$ & $\mathrm{D}_{4}$ & $\mathrm{D}_{4}$ \\
4 & $\mathrm{D}_{2}$ & $\mathrm{D}_{2}$ & $\mathrm{D}_{2}$ & $\mathrm{D}_{2}$ & $\mathrm{D}_{2}$ & $\mathrm{D}_{2}$ & $\mathrm{D}_{7}$ & $\mathrm{D}_{8}$ & $\mathrm{D}_{7}$ & $\mathrm{D}_{7}$ & $\mathrm{D}_{7}$ & $\mathrm{D}_{7}$ \\
5 & $\mathrm{D}_{4}$ & $\mathrm{D}_{4}$ & $\mathrm{D}_{7}$ & $\mathrm{D}_{7}$ & $\mathrm{D}_{4}$ & $\mathrm{D}_{4}$ & $\mathrm{D}_{9}$ & $\mathrm{D}_{9}$ & & $\mathrm{D}_{9}$ & $\mathrm{D}_{8}$ & $\mathrm{D}_{9}$ \\
6 & $\mathrm{D}_{11}$ & $\mathrm{D}_{11}$ & $\mathrm{D}_{13}$ & $\mathrm{D}_{13}$ & $\mathrm{D}_{11}$ & $\mathrm{D}_{11}$ & $\mathrm{D}_{11}$ & $\mathrm{D}_{11}$ & $\mathrm{D}_{11}$ & $\mathrm{D}_{11}$ & $\mathrm{D}_{11}$ & $\mathrm{D}_{11}$ \\
7 & $\mathrm{D}_{18}$ & $\mathrm{D}_{21}$ & $\mathrm{D}_{20}$ & $\mathrm{D}_{18}$ & $\mathrm{D}_{21}$ & $\mathrm{D}_{14}$ & $\mathrm{D}_{14}$ & $\mathrm{D}_{15}$ & $\mathrm{D}_{14}$ & $\mathrm{D}_{14}$ & $\mathrm{D}_{14}$ \\
8 & $\mathrm{D}_{28}$ & $\mathrm{D}_{32}$ & $\mathrm{D}_{30}$ & $\mathrm{D}_{27}$ & $\mathrm{D}_{32}$ & $\mathrm{D}_{31}$ & $\mathrm{D}_{17}$ & $\mathrm{D}_{17}$ & $\mathrm{D}_{17}$ & $\mathrm{D}_{18}$ & $\mathrm{D}_{18}$ & $\mathrm{D}_{17}$ \\
9 & $\mathrm{D}_{49}$ & $\mathrm{D}_{45}$ & $\mathrm{D}_{48}$ & $\mathrm{D}_{44}$ & $\mathrm{D}_{44}$ & $\mathrm{D}_{29}$ & $\mathrm{D}_{30}$ & $\mathrm{D}_{29}$ & $\mathrm{D}_{32}$ & $\mathrm{D}_{31}$ & $\mathrm{D}_{29}$ \\
10 & $\mathrm{D}_{60}$ & $\mathrm{D}_{60}$ & $\mathrm{D}_{62}$ & $\mathrm{D}_{62}$ & $\mathrm{D}_{64}$ & $\mathrm{D}_{59}$ & $\mathrm{D}_{49}$ & $\mathrm{D}_{47}$ & $\mathrm{D}_{45}$ & $\mathrm{D}_{46}$ & $\mathrm{D}_{45}$ & $\mathrm{D}_{43}$ \\
11 & $\mathrm{D}_{74}$ & $\mathrm{D}_{74}$ & $\mathrm{D}_{76}$ & $\mathrm{D}_{79}$ & $\mathrm{D}_{74}$ & $\mathrm{D}_{74}$ & $\mathrm{D}_{57}$ & $\mathrm{D}_{60}$ & $\mathrm{D}_{59}$ & $\mathrm{D}_{60}$ & $\mathrm{D}_{60}$ & $\mathrm{D}_{57}$ \\
\hline
\end{tabular}

Fig. 2. Planning of data measurements during the clinical trial. $\mathrm{D}_{0}$ is the first day of the trial for the patient. $\mathrm{D}_{i}$ is equal to the day $\mathrm{D}_{0}+i$.

protocol. Thus, the 11 blood samples of each patient have been kept frozen (at $-80^{\circ} \mathrm{C}$ ) until the end of the trial. The measurements were done at the same time using the Roche Taqman $48^{\mathbf{T M}}$ test, with a detectability threshold of 50 copies/ml. 


\section{Results}

In this section identification of the parameters of model (2) is performed, and, in particular, the identification of parameter $A$. Recall that life-time parameters are set to inter-patient constant values: $\delta=0.01 \mathrm{day}^{-1}, \mu=0.05 \mathrm{day}^{-1}, c=$ $0.3 \mathrm{day}^{-1}$. To our best knowledge on parameter uncertainties of the HIV/AIDS infection (Wei, L. et al. (1995), Ho, D.D et al. (1995), Table 2.1 of Perelson, A.S. et al. (1999), Ouattara, D. (2005)), parameter $c$ ranges in the interval $[0.1 ; 0.65]$ with a mean value close to $0.3 d_{a y}{ }^{-1}$. From the EDV05 results (when not fixing any parameter), $c$ ranges approximately in the interval $[0.1 ; 0.3]$ with a median 1 at $0.28 d a y^{-1}$. The results of the identification of $\mu$ and $\delta$ in previous studies were obtained based on the same Monte-Carlo approach described above. They suggest that $\mu$ has a median close to $0.05 d a y^{-1}$ and $\delta$ has a median close to $0.01 \mathrm{day}^{-1}$. Also, parameter $s$ is fixed at $6 C D 4 \mathrm{~mm}^{-3} \mathrm{day}^{-1}$. This reduces to the assumption that the thymus of all patients is healthy. Parameters $\beta$ and $k$ determine the virulence of the infection and include treatment's effect; thus, they don't reflect any property of the immune system. So, the only varying parameter which reflects the immune system's status is $A$.

In particular, identification was performed using only the first six blood samples for each patient. Indeed, the goal is to be able to diagnose immunological failure as soon as possible after starting a new therapy. The inclusion of the transient response samples is essential for an accurate identification of the parameters whereas the last blood samples typically do not contain significant additional dynamical information. However, the identification from the whole set of blood samples available has also been performed and the corresponding results are in accordance with those obtained using the first six blood samples only.

Results are listed in Table 2 which shows that patients 03, 07 and 11 have the highest values of parameter $A$. Furthermore, the interquartile range for parameter $A(\operatorname{IQR}(A))$ for these patients is almost isolated from the $\operatorname{IQR}(A)$ of other patients.

In fact, the immunological failure of patient 03 was clinically confirmed after 3 months of monitoring. From a clinical point of view, the first six data points for patient 07 suggest that he was also going through immunological failure, but starting $\mathrm{D}_{30}$ he entered a non-observance phase. Furthermore, no monitoring of this patient was possible after the end of the trial. Thus, the decision about the status of patient 07 remains controversial. On another hand, patient 11 was 59 years old at the trial time and is the oldest among the 10 patients which have been included. In this special case, a high value of the apoptosis parame-

1 The "median" is used instead of the "mean" since it is a more robust statistical criterion than mean with respect to noise in the data. 
ter rather indicates poor dynamics of the immune system which is standard at the age of 60. No immunological failure was observed for the 9 other patients. Immunological failure is predicted after less than 3 weeks of monitoring as displayed in Table 2. In this case, the effect of activation-induced apoptosis is detrimental to the immune system since the high apoptosis rate results in the auto-elimination of many healthy CD4 + T-cells. For the other patients, $A$ is too small to have a consequent effect on the immune system. Note that the model has no inter-patient prediction ability since parameters identification is derived for each patient alone and based on his own clinical data. The conclusion of table 2 is that the identification of the apoptosis parameter in model (2) gives a hint for the diagnosis of immunological failure. The pecularities of each patient such as therapy adherence or age have nevertheless to be considered afterwards to make the final clinical decision.

\begin{tabular}{c||cccc}
\hline Pat. ID & $\beta$ & $k$ & $\mathbf{A}$ & $I Q R(A)$ \\
\hline 01 & $1.94 \mathrm{E}-07$ & 0.04 & $\mathbf{5 . 2 6 E - 0 8}$ & {$[2.55 E-09 ; 1.58 E-04]$} \\
02 & $3.27 \mathrm{E}-07$ & 0.21 & $\mathbf{1 . 1 7 E - 0 7}$ & {$[4.05 E-09 ; 7.36 E-05]$} \\
03 & $8.24 \mathrm{E}-07$ & 0.41 & $\mathbf{1 . 2 5 E}-03$ & {$[2.80 E-04 ; 9.43 E-03]$} \\
04 & $7.10 \mathrm{E}-08$ & 293.00 & $\mathbf{7 . 6 6 E - 0 8}$ & {$[4.19 E-09 ; 2.77 E-05]$} \\
05 & $3.94 \mathrm{E}-07$ & 0.004 & $\mathbf{6 . 2 4 E - 0 6}$ & {$[3.44 E-09 ; 4.81 E-03]$} \\
06 & $1.21 \mathrm{E}-07$ & 46.80 & $\mathbf{4 . 2 2 E - 0 7}$ & {$[7.91 E-09 ; 5.14 E-04]$} \\
07 & $2.43 \mathrm{E}-06$ & 0.008 & $\mathbf{6 . 5 1 E - 0 3}$ & {$[4.41 E-03 ; 2.72 E-02]$} \\
08 & $8.15 \mathrm{E}-07$ & 0.002 & $\mathbf{5 . 1 7 E - 0 8}$ & {$[2.07 E-09 ; 7.13 E-04]$} \\
09 & $7.23 \mathrm{E}-07$ & 0.003 & $\mathbf{1 . 0 1 E - 0 5}$ & {$[5.52 E-09 ; 1.58 E-03]$} \\
10 & $6.16 \mathrm{E}-07$ & 0.008 & $\mathbf{2 . 4 4 E - 0 6}$ & {$[3.63 E-09 ; 1.61 E-03]$} \\
11 & $1.79 \mathrm{E}-07$ & 0.012 & $\mathbf{9 . 2 6 E - 0 3}$ & {$[8.42 E-03 ; 1.71 E-02]$} \\
12 & $2.56 \mathrm{E}-07$ & 0.002 & $\mathbf{2 . 4 9 E}-06$ & {$[2.21 E-08 ; 1.04 E-03]$} \\
\hline
\end{tabular}

Model 3DA parameters of the 12 patients of the EDV05 trial. Estimation done with the first 6 blood samples with $s=6 C D 4 m^{-3} d^{-1}$.

Results of the $4 D A$ model

Even though similar results are obtained when using models (2) and (3), numerical results of model (3) are not explicitly depicted in this paper. In fact, the amount of specific-HIV CTL lymphocytes is not measurable in practice. Indeed, the measured CTL count is the total amount of CTL cells in the plasma which may not be fully due to the HIV infection as it typically also includes 
the CTL cells due to other infections. In addition, for a fixed amount of data points, identification of a higher order system with additional parameters is typically less accurate than identification of the basic three dimensional model. For the above reasons, identification of the model (3) is not fully tractable and is not presented here.

\section{Conclusion}

In this paper, the influence of activation-induced apoptosis on the HIV dynamics was studied and it was shown that activation-induced apoptosis may characterize immunological failure depending on its intensity. The two main contributions are as follows. First, the activation-induced apoptosis phenomenon was incorporated into the basic 3D model of the HIV infection (Section 2.2). Second, it was shown that identification of the apoptosis parameter based on ad hoc clinical data allows to predict the immunological failure of patients (Section 5). These results could also help in designing new anti-HIV therapies based on the regulation of activation-induced apoptosis factors in HIV infected patients. These therapies could include some interleukins such as IL-2, IL-7 and IL-15 whose anti-apoptotic effect has been reported (Ahr, B. et al. (2004), Vassena, L. et al. (2007)). The idea of studying the apoptosis phenomenon was suggested by recent research papers relating the decline of CD4+ T-cells to programmed cells death (Pantaleo, G. et al. (1995), Herbein, G. et al. (1998), Marie-Lise Gougeon et al. (1999), Ahr, B. et al. (2004), Badley, A.D. (2005), Yun Yue, F. et al. (2005), Stan, G.B. et al. (2008), Vassena, L. et al. (2007)). After testing the identifiability of the newly apoptosis compliant model and explaining the estimation procedure of the parameters, it was shown that the so called apoptosis parameter identified from real clinical data is a good alternative predictor of immunological failure. Previous studies (Ouattara, D. (2005), Ouattara, D. (2007), Ouattara, D. et al. (2008)) relate the decline of the $\mathrm{CD} 4+\mathrm{T}$-cells to a low production rate of uninfected cells due to a damaged thymus. Whether immunological failure is predominantly due to a badly damaged thymus or to an important activation-induced apoptosis phenomenon remains an open question. Further research including clinical data that evaluates the thymus status for HIV infected patients may help in understanding the real reason behind the depletion of CD4 + T-cells.

\section{Acknowledgments}

The EDV05 clinical trial has been performed with the financial support of the CHU of Nantes (Nantes University Hospital). The second author is a research 
associate of the Belgian FNRS whose financial support is acknowledged. The last author is a research associate with financial support from United Kingdom EPSRC under grant No. EP/E02761X/1. The third author thanks the Belgian Network BIOMAGNET (Bioinformatics and Modeling: from Genome to Networks).

\section{References}

Adams, B.M and Banks, H.T. and Hee-Dae Kwon and Hien T. Tran, Dynamic multidrug therapies for HIV: Optimal and STI approaches, Mathematical Biosciences and Engineering,1(2), September 2004.

Ahr, B. and Robert-Hebmann, V. and Devaux, C. and Biard-Piechaczyk, M., Apoptosis of uninfected cells induced by HIV envelope glycoproteins, Retrovirology, (1), june, 2004.

Andrew D. Badley ,2005, Cell death during HIV infection, CRC Press, URL http://www.vonl.com/chips/cellhiv.htm.

Berger A. et al., 2005, Comparative evaluation of the COBAS Amplicor HIV-1 Monitor $^{T M}$ Ultrasensitive Test, the new COBAS AmpliPrep/COBAS Amplicor HIV-1 Monitor ${ }^{T M}$ and the Versant HIV RNA 3.0 assays for quantitation of HIV-1 RNA in plasma samples, Journal of Clinical Virology, 33, $43-51$.

Chang, H. Astolfi, A., Immune response's enhancement via controlled drug scheduling, 46th IEEE Conference on Decision and Control, December 2007, New Orleans, LA, USA.

Delfraissy JF., Prise en charge des personnes infectées par le VIH : Recommandations du groupe d'experts, Flammarion edition, Médecine Science, Paris, 2005, Available at www.ladocumentationfrancaise.fr/brp/notices/044000467.shtml.

Estaquier, J. and others, Programmed cell death and AIDS: Significance of Tcell apoptosis in pathogenic and nonpathogenic primate lentiviral infections, Proc. Natl. Acad. Sci. USA, (91), 9431-9435, September 1994.

Fiches Techniques de la Firme Roche, 2003,Available at http://www.roche-diagnostics.fr.

Filter, R.A. and Xia, X., A penalty function to HIV/AIDS model parameter estimation, 13th IFAC Symposium on System Identification, Rotterdam, 2003.

Galli, R., Merrick, L., Friesenhahn, M., Ziermann, R.,2005, Comprehensive comparison of the Versant ${ }^{\circledR}$ HIV-1 RNA 3.0 (bDNA) and COBAS Amplicor HIV-1 Monitor ${ }^{\circledR} 1.5$ assays on 1000 clinical specimens, Journal of Clinical Virology, 34, 245-252.

Gougeon, M-L. and Montagnier,L., 1999, Programmed Cell Death as a Mechanism of CD4 and CD8 T Cell Depletion in AIDS: Molecular Control and Effect of Highly Active Anti-Retroviral Therapy, Annals of the 
New York Academy of Sciences, 887 (1), 199-212, http://www.blackwellsynergy.com/doi/abs/10.1111/j.1749-6632.1999.tb07934.x.

Heffernan, J.M and Wahl, L.M, Treatment interruptions and resistance: a review, In Deterministic and stochastic models for AIDS epidemics and HIV infection with interventions (ed. W.-Y. Tan and H. Wu),425-455, Hackensack, NJ: World Scientific.

Herbein, G., Mahlknecht, U., Batliwalla, F., Gregersen, P., Pappas, T., Butler, J., O'Brien, W.A., Verdin, E., 1998, Apoptosis of CD $8+$ T cells is mediated by macrophages through interaction of HIV gp120 with chemokine receptor CXCR4, Nature, 395 (6698), 189-194, URL http://dx.doi.org/10.1038/26026.

Ho, David D. et al., 1995, Rapid turnover of plasma virion and CD4 lymphocytes in HIV-1 infection, Nature, 373, 123-126.

Israel-Ballard, K. et al., 2005, TaqMan RT-PCR and Versant ${ }^{\circledR}$ HIV-1 RNA 3.0 (bDNA) assay Quantification of HIV-1 RNA viral load in breast milk, Journal of Clinical Virology, 34, 253-256.

Khalili, S. and Armaou, A., Sensitivity analysis of HIV infection response to treatment via stochastic modeling, Chemical Engineering Science, (63), 1330-1340, 2008.

Moog,C.H, Ouattara, D.A and Mhawej,M.J, Analysis of the HIV dynamics, Proceedings of the 7th IFAC Symposium on Nonlinear Control Systems, August 22-24,2007, Pretoria, South Africa.

Nowak, M.A. and May, R.M., 2002, Virus dynamics: Mathematical principles of immunology and virology, Oxford University Press.

Ouattara, D.A., Mathematical Analysis of the HIV-1 Infection: Parameter Estimation, Therapies Effectiveness, and Therapeutical Failures, Sep.2005, 27th Annual International Conference of the IEEE Engineering in Medecine and Biology Society, Shanghai, China.

Ouattara, D.A., Sep.2006, Modélisation de l'infection par le VIH, identification et aide au diagnostic, $\mathrm{PhD}$ Thesis, Ecole Centrale de Nantes \& Université de Nantes, Nantes, France.

Ouattara, D.A. and Mhawej,M.-J. and Moog, C.H., IRCCyN Web software for the computation of HIV infection parameters, Available at http://www.hiv.irccyn.ec-nantes.fr, 2007.

Ouattara, D.A. and Moog, C.H., 2007, Modelling of the HIV/AIDS infection : an aid for an early diagnosis of patients, Biology and control theory: current challenges, Springer Verlag, Springer Series in Lecture Notes in Control and Information Sciences (LNCIS).

Ouattara, D.A and Mhawej, M.J and Moog, C., Clinical tests of therapeutical failures based on mathematical modeling of the HIV infection, Joint special issue of IEEE transactions on circuits and systems and IEEE transactions on Automatic Control, Special issue on systems biology, 230-241, January 2008.

Pantaleo, G., Fauci, A.S.,Feb.1995, Apoptosis in HIV infection, Nature Medicine, 1 (2), 118-120, URL http://dx.doi.org/10.1038/nm0295-118. 
Perelson, A.S. and Nelson, P.W., 1999, Mathematical analysis of HIV-1 dynamics in vivo, SIAM Review, 41 (1), 3-44.

Perelson, A.S. et al., 1997, Decay characteristics of HIV-1 infected compartment during combination therapy, Nature, 387, 188-191.

Prud'homme, I.T. et al., 1998, Amplicor HIV Monitor, NASBA HIV-1 RNA QT and Quantiplex HIV RNA version 2.0 viral load assays: a Canadian evaluation, Journal of Clinical Virology, 11, 189-202.

Smith R.J., Adherence to antiretroviral HIV drugs: how many doses can you miss before resistance emerges?, Proceedings of the Royal Society B, 273, 617-624, 2006.

Smith, R.J. and Wahl, M., Distinct effects of protease and reverse transcriptase inhibition in an immunological model of HIV-1 infection with impulsive drug effects, Bulletin of Mathematical Biology, (66), 1259-1283, 2004.

Stan, G.B, Belmudes, F., Fonteneau,R., Zeggwagh, F., Lefebvre, M.A, Michelet, C., Ernst, D., 2007, Modelling the influence of activation-induced apoptosis of $\mathrm{CD} 4+$ and $\mathrm{CD} 8+\mathrm{T}$-cells on the immune system response of a HIV infected patient, IET Systems Biology, Vol 2, Issue 2, 94-102, 2008.

Stewart S.A., and Poon B., and Song J.Y., and Chen, I.S.Y., Human Immunodeficiency Virus Type 1 Vpr Induces Apoptosis through Caspase Activation, The Journal of Virology, (74), 3105-3111, April 2000.

Tan, W.Y and Wu, H., Deterministic and stochastic models for AIDS epidemics and HIV infection with interventions, World Scientific, 2005.

U.S. Dept. Health and Human Services, May 2006, Guidelines for the Use of Antiretroviral Agents in HIV-1-Infected Adults and Adolescents, Available at http://www.aidsinfo.nih.gov/guidelines.

Vassena,L., Proschan, M., Fauci, A.S and Lusso P., 2007, Interleukin 7 reduces the level of spontaneous apoptosis in CD4 + and CD $8+\mathrm{T}$ cells from HIV-1 infected individuals, Proceedings of the National Academy of Sciences of the United States of America, 2355-2360.

Wang, J., and Guan, E., and Roderiquez, G. and Norcross, M.A., Synergistic Induction of Apoptosis in Primary CD4 $+\mathrm{T}$ Cells by Macrophage-Tropic HIV-1 and TGF-beta1, The Journal of Immunology, (167), 3360-3366 , September 2001.

Wei, X. et al., Jan. 1995, Viral dynamics in Human Immunodeficiency virus type 1 infection, Nature, 373,117-122.

Wodarz, D. and Nowak, M., Specific therapy regimes could lead to long term immunological control of HIV, Proceedings of the National Academy of Sciences of the United States of America (PNAS), 96(25),14464-14469, December 1999.

Xia, X. and Moog,C.H., Feb. 2003, Identifiability of Nonlinear Systems with Application to HIV/AIDS Models, IEEE Transactions on Automatic Control, 48 (2), 330-336.

Yun Yue,F., Kovacs, C., Dimayuga, R. Xiao, J.G., Parks,P., Kaul, R., Ostrowski, M., 2005, Preferencial apoptosis of HIV-1 Specific CD4 + T cells, The journal of immunology, 174, 2196-2204. 
Zauli, G., Gibellini, D., Secchiero P., Dutartre, H., Olive, D., Capitani, S., and Collette, Y., Human Immunodeficiency Virus Type 1 Nef Protein Sensitizes CD4 + T Lymphoid Cells to Apoptosis via Functional Upregulation of the CD95/CD95 Ligand Pathway, Blood, (93),1000-1010, February 1999.

Zurakowski, R. and Teel, A., Enhancing immune response to HIV infection using MPC-based treatment scheduling, American Control Conference, June 4-6, 2003, Denver, Colorado.

Zurakowski, R. and Teel, R.A., A model predictive control based scheduling method for HIV therapy, Journal of Theoretical Biology, 368-382, July 2006.

Zurakowski, R. and Wodarz, D., Treatment interruptions to decrease risk of resistance emerging during therapy switching in HIV treatment, 46th IEEE Conference on Decision and Control, December 2007, New Orleans, LA, USA. 